Revista de Psicología Vol. 36 (2), 2018 (ISSN 0254-9247)

\title{
Adaptación de la Escala de Percepción Global de Estrés en estudiantes universitarios peruanos
}

\author{
Jaime Enrique Guzmán-Yacaman ${ }^{1}$, Mario Reyes-Bossio ${ }^{2}$ \\ Universidad Peruana de Ciencias Aplicadas - UPC
}

\begin{abstract}
El objetivo del presente estudio consistió en validar la Escala de Percepción Global de Estrés en una muestra peruana de universitarios de un programa nacional de becas. El proceso de adaptación consideró la doble traducción a partir de la versión original, la comparación lingüística con la adaptación chilena y mexicana, así como la revisión por jueces. Asimismo, se aplicó una prueba piloto. Los participantes fueron 332 becarios universitarios. Los resultados del Análisis Factorial Confirmatorio confirmaron el modelo bifactorial de la EPGE-13. Asimismo, se obtuvieron evidencias de validez divergente y convergente utilizando el SPANAS. La confiabilidad estimada de los puntajes generados por el instrumento fueron $\alpha=.79$ para el factor de eustrés y $\alpha=.77$ para el factor de distrés. Los resultados demográficos indicaron mayor presencia de estrés percibido en mujeres que en hombres. No se observaron diferencias entre el estrés y el lugar de procedencia.

Palabras clave: estrés académico, afecto, validez, confiabilidad, estudiantes universitarios.
\end{abstract}

\section{Adaptation of the Global Perceived Stress Scale in college peruvian students}

The present study aims to validate the Global Perceived Stress Scale on a Peruvian college students sample, involving young scholars of a state-funded program. The adaptation process involved a double translation from the original version, a linguistic comparison using the scales adapted in Chile and Mexico, and judgement criteria, as well as a pilot test. Participants were 332 college scholarship students. Results with the Confirmed Factorial Analysis confirmed the bifactorial model of the EPGE-13. Moreover, convergent and divergent validity was done with the SPANAS test. Likewise, the scale demonstrate adequate reliability (internal consistency, $\alpha=.79$ for the factor of eustress and $\alpha=.77$ for the factor of distress). Demographic results indicated that there was more perceived stress in women than in men. There were no significant differences in stress according to birthplace.

1 Licenciado en Psicología y analista de Orientación Psicopedagógica de Calidad Educativa de la UPC. Dirección postal: Universidad Peruana de Ciencias Aplicadas - UPC, Lima Perú. Contacto: jaime.guzman@upc.pe; j_guzman4@hotmail.com

2 Magíster en Psicología y docente de la Facultad de Psicología de la UPC. Dirección postal: Universidad Peruana de Ciencias Aplicadas - UPC, Lima Perú. Contacto: mario.reyes@upc. pe; marb2383@hotmail.com 
Keywords: Academic Stress, affection, Statistical Validity, Statistical Reliability, College Students.

\begin{abstract}
Adaptaçáo da Escala Global de Percepçáo de Estresse en estudantes universitarios peruanos

O objetivo deste estudo foi validar a Escala Global de Percepção de Estresse numa amostra peruana de estudantes um programa nacional de bolsas de estudo. O processo de adaptação considerou a dupla tradução da versão original, a comparação linguística com a adaptação chilena e mexicana, como a revisão pelos revisores. Assim mesmo, um teste piloto foi realizado para ser aplicado posteriormente aos 332 universitários bolsistas que participaron neste estudo. Os resultados da Análise Fatorial Confirmatória (AFC) confirmaram o modelo bifatorial da EPGE-13. As evidências de validade divergente e convergente foram obtidas usando o SPANAS. A confiabilidade estimada dos escores gerados pelo instrumento foi $\alpha=.79$ para o fator "eutrés" e $\alpha=.77$ para o fator de "distrés". Os resultados demográficos indicaram uma maior presença de estresse percebido nas mulheres que nos homens. Porém, não houve diferenças significativas entre estresse e local de origem.

Palavras-chave: estresse acadêmico, afetos, validade, confiabilidade, estudantes universitários.
\end{abstract}

\title{
Adaptation de l'échelle globale de perception du stress chez les étudiants universitaires péruviens
}

L'objectif de cette étude était de valider l'échelle globale de perception du stress dans un échantillon péruvien d'étudiants universitaires du un programme de bourses d'études national. Le processus d'adaptation a pris en compte la double traduction de la version originale, la comparaison linguistique avec l'adaptation chilienne et mexicaine, ainsi que l'examen par les juges. Un essai pilote a également été réalisé et appliqué aux participants de cette étude. Les participants étaient 332 étudiants boursiers. Les résultats de l'analyse factorielle ont confirmé le modèle bifactoriel de l'EPGE-13. Des preuves de validité divergente et convergente ont également été obtenues à l'aide de SPANAS. La fiabilité estimée des scores générés par l'instrument était $\alpha=.79$ pour le facteur eustress et $\alpha=.77$ pour le facteur stress négatif. Les résultats démographiques indiquent une présence plus élevée de stress ressenti chez les femmes que chez les hommes. Aucune différence significative n'a été observée entre le stress et le lieu d'origine.

Mots clés: Stress académique, affection, validité statistique, fiabilité statistique, Étudiants universitaires. 
El estrés, según Selye (1956, citado en Pulido et al., 2011), es una respuesta adaptativa que el organismo humano tiende a presentar ante la necesidad de habituarse a una situación o entorno nuevo. El desarrollo teórico de este término ha ido evolucionando a lo largo del tiempo, pasando de un esquema estímulo-respuesta a un esquema dinámico y persona-entorno (Trianes, Blanca, Fernández-Baena, Escobar \& Maldonado, 2012). A partir de esta nueva concepción teórica del estrés, se ha visto la necesidad de estudiarlo en la persona a partir de su interacción con su entorno más próximo y ver si en él se dan patrones cuya situación fomenta su aparición en los seres humanos. Siguiendo esta línea, uno de los campos en donde más predomina o tiende a predominar el estrés es en el de la educación (Barraza, 2006; Barraza, Carrasco \& Arreola, 2009; González \& Landero, 2007a). En él se promueve un escenario prometedor para el estudio del estrés en las personas, haciéndose llamar estrés académico.

Para Pulido et al. (2011), el estrés académico está presente en estudiantes de primaria y secundaria, sin embargo, se agudiza aún más cuando se da la transición de los estudios secundarios a los universitarios. Este fenómeno surge en el alumno, debido a que comienza a sobrellevar nuevas situaciones poco habituales como las altas cargas de trabajo, nuevos círculos a los cuales adaptarse, mayor separación del seno familiar, adquisición de más independencia o una posible incorporación al mercado laboral para poder cubrir sus estudios. Asimismo, constituye una experiencia de sobreadaptación, requiriendo que la persona entre en una reorganización de las áreas que giran en torno al aprendizaje y nuevas metodologías de estudio o áreas en relación a lo intra e interpersonal, que buscan generar nuevas redes de apoyo social.

Otros estudios postulan que los altos niveles de estrés son atribuidos a la exposición a trabajos en el salón de clase, la falta de tiempo para realizar las tareas de acuerdo al cronograma, la sobrecarga de clases 
en la malla curricular y la realización de evaluaciones (García-Ros, Pérez-González, Pérez-Blasco \& Natividad, 2011).

Gallardo y Morales (2011) comentan que estas situaciones adversas tienen que ver con temas en base a su identidad: la forma en la que ponen a prueba su autoestima, evalúan su proyecto de vida, crean nuevas redes de amistades e intimidad, balancean la actividad académica con las personales y practican formas más complejas de pensamiento. De no saber cómo afrontarlas adecuadamente se vuelven propensos a desencadenar factores de estrés académico, generando en él un desequilibrio. Esto puede ser comprendido a partir del supuesto cognoscitivista o transaccional de estrés (Barraza, 2006).

El supuesto cognoscitivista o teoría transaccional del estrés, se preocupa por lo que sucede en la persona cuando este recepciona información de su entorno. Esta perspectiva cognoscitiva creada por Lazarus y Folkman (1986) fue utilizada por Cohen, Kamarck y Mermelstein (1983) en la construcción de la Escala de Percepción Global de Estrés. En él se busca explicar la manera en la que la persona en transacción continua con su entorno, tiende a valorar las demandas que este infringe sobre él como amenazantes o difíciles de afrontar.

Por su parte, Lazarus y Folkman (1986) proponen tres tipos de evaluación: en la primera evaluación se da el encuentro con algún tipo de demanda tanto interna como externa, para luego, en una segunda instancia, valorar los propios recursos para afrontar y responder ante el estímulo estresor; estas valoraciones pueden catalogarse como neutra, positiva o negativa. Finalmente, cuando no existe un equilibrio entre los acontecimientos catalogados como estresante y los recursos disponibles aparece el estrés que genera que el alumno utilice diferentes estrategias de afrontamiento a partir del problema (controlar la situación causante de estrés) y sobre la base de la emoción, intentando regular la respuesta emocional (Barraza, 2006). Del adecuado proceso que se lleve a cabo en esta transacción, depende si se da un estrés positivo o eustrés, que se produce cuando los recursos físicos y psicológicos de la persona son adecuados con relación a la demanda; o un estrés negativo o distrés, 
que se da cuando las respuestas han sido insuficientes en relación con la demanda del entorno (Berrio \& Mazo, 2011).

\section{Pobreza como variable estresora del estrés académico}

La condición de pobreza durante la vida de una persona llega a afectar al desarrollo cognitivo, socioemocional y afectivo, lo que los expone a ser más vulnerables a presentar patrones de estrés psicológico, disminuyendo así, la capacidad para adoptar patrones más adecuados de interacción con su entorno y de resolución de problema-adaptación (Emerson, 2004; Lera, 2009). De igual modo Wadsworth, Raviv, Reinhard, Wolff, Santiago y Einhorn (2008) postula que si la pobreza se ha desarrollado de manera crónica en la persona, se puede llegar a acarrear un efecto negativo en cadena, haciendo que la persona se se enfoque en toda una serie de episodios estresantes, ocasionando la falta de recursos personales para la solución de conflictos, contribuyendo en el aumento del estrés y la aparición de cuadros depresivos.

Según estudios del Instituto Nacional de Estadística e Informática (2015) el 23.9\% de la población total se encuentra en calidad de pobreza extrema monetaria, siendo el área rural la más afectada. Es por eso que a inicios del ańo 2012 el Programa Nacional de Becas y Crédito Educativo, a través del Ministerio de Educación, implementó Beca 18. El objetivo de este programa es mejorar la equidad en el acceso de la educación superior, financiando todos los costos directos e indirecto desde el inicio de las clases y durante todos los estudios de pregrado a jóvenes de escasos recursos. Anualmente, este gran proyecto, subvenciona un promedio de 3.500 alumnos de escasos recursos de todo el Perú que ingresan a 17 universidades y 12 institutos (Programa Nacional de Becas y Crédito, 2014).

A partir de este respaldo de corte social, los jóvenes se ven menos vulnerables ante el impacto de sus antecedentes socioeconómicos, facilitándoles desarrollar su resiliencia. Sin embargo, la ayuda social no es la única fuente generadora de resiliencia en la persona, ya que según estudios de Garmezy (1993) y Richardson (2002), la resiliencia ante 
situaciones cargadas de estresores en personas con antecedentes de pobreza, resulta a partir de la complementación de un modelo de tres factores principales. En este modelo intervienen la parte social, aspectos familiares y factores personales; son en estos dos últimos donde también se deben realizar intervenciones, debido a la siguiente razón: al contar con un soporte familiar, el alumno tiene un sentido de seguridad en casa, muestras de afecto o presencia de algún adulto que los cuida (Garmezy, 1993). No obstante la gran mayoría de los alumnos becarios vienen del interior del país y viven solos en complejos habitacionales o departamentos compartidos que el estado les facilita. Asimismo, en base a los factores personales sabemos que estos jóvenes, por los ya mencionados antecedentes de pobreza y un carente lugar para desarrollar la resiliencia, pueden presentar dificultades en campos como su autoestima, percepción de autoeficacia, expectavivas de éxito, optimismo y esperanza (Rutter, 1987), los cuales llegan a impactar en su desenvolvimiento académico. Cabe resaltar que a este modelo se agrega el factor psicosocial que puede llegar a ser un generador de estrés, debido al cambio de localidad, costumbres y cultura que sufren estos alumnos al migrar del interior del país a un lugar nuevo y poco conocido como lo es la ciudad de Lima (Achotegui, 2010).

Por lo tanto, es necesario reforzar el área de intervención psicológica del programa a partir del trabajo en las áreas mencionadas, para impedir el desencadenamiento de un cuadro de estrés negativo, y asegurar en los alumnos un óptimo desempeño académico, evitando una posible deserción. Para esta razón, es importante contar con instrumentos psicométricos como la Escala de Percepción Global de Estrés (EPGE) que brinda una mejor perspectiva del estrés negativo, pero también brinda información de las conductas adecuadas o adaptativas que tanto necesitan los alumnos para sobrellevar una situación con estresores.

De esta manera la EPGE puede cumplir con la misión de brindar un mayor refuerzo en el área de seguimiento de los becarios y monitoreo institucional, para analizar qué situaciones pueden significar riesgos que generen fracaso académico o deserción de la carrera. Asimismo, este instrumento pemite un mayor acercamiento personalizado a los 
becarios para poder abordar todo tipo de afección que atente contra su bienestar personal y psicológico. De esta manera se complementa la labor realizada por Alerta Becario -Equipo que brinda un servicio virtual de orientación y consejería especializada en salud mental a nivel nacional a los becarios del programa Beca 18-, que si bien se encarga de abordar áreas en relación al soporte emocional del alumno, puede tener un mayor impacto en su labor fomentando una atención presencial y mejor focalizada en la afección psíquica del alumno.

Por otro lado, el valor teórico de un instrumento como la EPGE adaptada se centra en estar desarrollada bajo la teoría transaccional del estrés y la colaboración de jueces expertos para la adaptación del instrumento. Finalmente, desde una perspectiva metodológica la EPGE dará a la ciencia, desde la psicología, un instrumento adaptado que permitirá entender los niveles del estrés positivo y negativo; a fín de generar investigaciones sobre este síndrome.

\section{Propiedades psicométricas de la EPGE-14}

La escala EPGE-14 es un instrumento de medición respecto al resultado de los niveles de estrés vivenciados por la persona en los últimos meses de vida (Cohen et al., 1983). La Escala está conformada por 14 ítems, de los cuales los ítems 4, 5, 6, 7, 9, 10 y 13 corresponden a la dimensión positiva o de eustrés y los ítems 1, 2, 3, 8, 11, 12 y 14 a la dimesión negativa o de distrés. Su puntaje global se obtiene mediante la suma entre la reversión de los puntajes de los ítems positivos (estos se caracterizan por tener un enunciado positivo) y los puntajes de los ítems negativos. Para la construcción de la escala se tuvo que evaluar tres muestras.

La primera muestra estaba conformada por 332 alumnos recién ingresantes a la Universidad de Oregón. La media de la edad era de 19.1. Se les aplicaron cinco escalas distintas, una de eventos significativos, la segunda de ansiedad, la tercera de sintomatología depresiva, la cuarta de sintomatología física y la última de la escala de estrés percibido. La duración de la evaluación de esta primera muestra duró 90 días. La segunda muestra fue de 114 alumnos (53 mujeres, 60 hombres y uno de sexo no 
especificado) que cursaban la clase introductoria de la personalidad en psicología. Su media de edad fue de 20.75 y se aplicaron las cinco escalas que se respondieron en la primera muestra. El trabajo con esta muestra duró un total 136 días entre evaluación y corrección.

La tercera muestra se realizó con un grupo de personas de un programa para disminuir el hábito tabáquico, en ella había 27 hombres y 37 mujeres que fueron solicitados a través del periódico para formar parte del programa que la Universidad de Oregón tenía para tratar a los fumadores que querían cesar de fumar. En la muestra la media de edad era de 38.4 ańos. $37 \%$ de la muestra obtenía un ingreso promedio de 25,000 dólares por año y $74 \%$ tenía una educación secundaria terminada. Se les aplicaron tres escalas: la de eventos significativos, la escala de chequeo de sintomatología física y la escala de percepción de estrés (Cohen et al., 1983).

A partir de las evidencias de validez, se utilizó una validez relacionada con criterios, dentro de la cual se optó por la concurrente. En los análisis de dicha validez ser reportaron correlaciones significativas con el CSLES ( $r=.30$ en estudiantes; $r=.49$ en fumadores); con síntomas depresivos ( $r=.76$ en estudiantes; $r=.65$ en fumadores); con la CHIPS $(r=.52$ en estudiantes; $r=.70$ en fumadores) y con el SADS $(r=.37$ en estudiantes; $r=.48$ en fumadores). Se encontró también que a mayor puntaje en la EPGE mayor consumo de cigarrillos habían tenido las personas del programa. La confiabilidad estimada de los puntajes generados por el instrumento reportaron coeficientes alfa de Cronbach de $.84, .85$, y .86 , en cada una de las tres muestras. Asimismo, la confiabilidad estimada de los puntajes generados por el instrumento reportaron coeficientes de estabilidad (test-retest) de $r=.85$ para la muestra de estudiantes con un intervalo de 2 días y de $r=.55$ para la muestra del programa de fumadores con un intervalo de seis semanas.

Adaptaciones asiáticas y europeas de la EPGE-14. En Asia la EPGE se ha adaptado en tres países. Dentro de ellos encontramos a China (Leung, Lam \& Chan, 2010), Japón (Mimura \& Griffiths, 2004) y Corea (Lee, Chung, Suh \& Jung, 2015). Sus trabajos de adaptación se pueden ver en el Anexo 1. En Europa, la escala se ha adaptado en 
Francia (Lesage, Berjot \& Deschamps, 2012), Grecia (Andreou et al., 2011) y Hungría (Stauder \& Konkoly, 2006). Dichos trabajos de adaptación se pueden ver explicados en el Anexo 2. En España, Remor (2006) realiza la primera adaptación de la Escala al idioma castellano, en su estudio utilizó una muestra de 440 participantes.

Para las evidencias de validez convergente se utilizó la escala de ansiedad y depresión en hospitales (HADS). En cuanto a la validez concurrente se realizó una correlación de Pearson entre las dos escalas. Los resultados indicaron que las medidas de ambos instrumentos utilizados, correlacionaban significativamente. Se asoció la ansiedad y la depresión hospitalaria con el estrés percibido en los pacientes $(r=.71$ y $r=.64$ respectivamente), determinándose de esta manera la sensibilidad de la escala al esta discriminar entre grupos de personas que denotan ansiedad y grupos que denotan estrés. En cuanto a sus resultados de confiabilidad se obtuvo un coeficiente alfa de Cronbach de .81. Luego después de dos semanas de haber aplicado el test se acudió nuevamente a un análisis test-retest donde la correlación fue $r=.73$.

EPGE adaptada en México. En Latinoamérica las adaptaciones como las de González y Landero (2007b) en México, tuvieron como propósito analizar la adaptación de la EPGE hecha por Remor (2006) para ser utilizadas en muestras mexicanas. Para ello se obtuvo una muestra de 365 participantes en donde había un 20\% de hombres y un $80 \%$ de mujeres con una media de edad de 20.48 ańos. Utilizaron tres instrumentos dentro de los cuales estaban: la EPGE, el inventario de Depresión de Beck y la Escala de Cansancio Emocional. Asimismo se efectuó una adaptación lingüística que indicó una modificación en la expresión castellana del ítem 12 .

Dentro de las propiedades psicométricas, se obtuvieron evidencias de validez externas. Para ello se llevó a cabo el análisis de validez convergente entre EPGE, el Inventario de Depresión de Beck y la Escala de cansancio emocional. Como se hipotetizó, ambas escalas se correlacionaron de manera positiva y significativa con la EPGE $(r=.55 \mathrm{y}$ $r=.52$, para el IDB y la ECE, respectivamente). Se realizó un Análisis Factorial Confirmatorio con el objetivo de corroborar el modelo de dos 
factores correlacionados que resultó en el Análisis Factorial Exploratorio (Los dos factores representaron el $48.02 \%$ de la varianza total, donde el primer factor explicaba el $32.61 \%$ y el segundo el $15.41 \%)$. Los indicadores de ajuste indicaron que el modelo era adecuado $\left(\chi^{2}(64)=\right.$ 200.979; RMSEA = .067; CFI = .92; GFI = .93). La confiabilidad de las puntuaciones mostraron coeficientes de consistencia interna alfa de Cronbach .83, para el factor de estrés negativo y .78 para el factor de estrés positivo.

EPGE adaptada en Venezuela. En Sudamérica, Benítez, D Anello y Noel (2012) comenzaron la adaptación de la EPGE en Venezuela. Contaron con una muestra no aleatoria de 867 funcionarios públicos con edades que oscilaban entre los 17 y 33 años. Los instrumentos utilizados fueron la EPGE, LEVENSON (Internalidad), NORMALTA (Ajuste psicológico), NR-21 (Psicológicamente bien consigo misma), el RESI (Resiliencia), ANSILET (Ansiedad) y GEdpr (Depresión). Cabe mencionar que para efectos de está adaptación se invirtieron los ítems negativos a positivos, por lo que a mayor puntaje menor estrés negativo.

Para determinar la dimensionalidad de la escala se hizo un análisis factorial de componentes principales en donde se reportó una estructura bifactorial. Para confirmar dicha estructura factorial se utilizó un modelo de ecuaciones estructurales (bifactorial), empleándose una segunda submuestra que representaba el $46 \%$ de la muestra total. A partir de ello se evidenció que el ítem 12 no obtuvo un coeficiente de regresión significativo, por lo que se procedió a su exclusión del modelo final, quedando así el factor negativo con 6 ítems y la escala total con 13 ítems.

Para efectos de la validez convergente y divergente la EPGE se correlacionó con 5 variables: Internabilidad, ajuste psicológico, resiliencia, ansiedad y depresión. Las correlaciones con la EPGE se presentaron según lo esperado $(r=.44 ; r=.26 ; r=.54 ; r=-.22$ y $r=-.12$, para Internalidad, Resiliencia, Ajuste psicológico, Ansiedad y Depresión, respectivamente). Para establecer la confiabilidad de las puntuaciones obtenidas del instrumento se calculó el alfa de Cronbach encontrando un valor de .95 para la versión final de 13 ítems. Se encontró, que las mujeres presentan mayores niveles de estrés percibido. 
EPGE adaptada en Chile. Tapia, Cruz, Gallardo y Dasso (2007) llevaron a cabo la adaptación de la EPGE en una población chilena de 117 alumnos mayores de 21 años. Inicialmente se realizó una validez de contenido en donde se discutieron las sugerencias brindadas por los jueces expertos, dando como resultado final la utilización de los 14 ítems de la escala. En la evidencias de validez concurrente se realizó un estudio correlacional entre el EPGE y el STAI, utilizando el coeficiente de correlación de Pearson entre los puntajes del EPGE y el STAI, lo cual dió como resultado una correlación positiva significativa $(r=.459$, $p=.001$ ).

Sobre la base de sus resultados se obtuvo un coeficiente alfa de Cronbach de .79 para el EPGE y de .94 para el inventario de Ansiedad de Spielberg siendo ambos de alta confiabilidad. Si bien la EPGE ha sido adaptada ya en varios paises de tres continentes, incluyendo paises sudamericanos, en el Perú aún no ha sido adaptada. Por ello el objetivo central de está investigación es adaptar la Escala de Percepción Global de Estrés a una muestra de alumnos universitarios del programa Beca 18 de los primeros ciclos. Todo ello mediante el reporte de las evidencias de validez de contenido y constructo, y el reporte de confiabilidad de las puntuaciones por medio del cálculo del coeficiente de consistencia interna alfa de Cronbach (Alarcón, 2013). Por esto el diseño de esta investigación es de tipo instrumental, ya que dicho estudio estará enfocado en la adaptación de una escala de medición psicológica que en este caso es la Escala de Percepción Global de Estrés (López, Ato \& Benavente, 2013).

\section{Método}

\section{Participantes}

La muestra estuvo constituida por 332 estudiantes, en donde el $51.20 \%(n=170)$ son mujeres y el $48.80 \%(n=162)$ son hombres y se encontraban matriculados en una universidad privada de Lima. El muestreo fue no probabilístico, intencionado, ya que se integró la 
muestra con individuos que se estiman como típicos o representativos de la muestra que se está buscando para fines de la investigación (Alarcón, 1991). El rango de edad oscila entre los 16 y 25 años $(M=17.76$, $D E=1.46)$. La mayoría de ellos proviene del interior del país, representando $78 \%$ de la muesta total $(n=259)$, mientras que el $22 \%(n=72)$ son de la ciudad de Lima. De la muestra el 31\% $(n=53)$ declararon que como curso más difícil es el de Lenguaje. En cuanto a criterios de inclusión se busca que los alumnos pertenezcan al programa de Beca 18, ya que dicho programa clasifica a los alumnos bajo los criterios pobreza o extrema problema y que estén actualmente matriculados en una universidad privada de Lima Metropolitana.

\section{Medición}

Para la medición del estrés se utilizó la Escala de Percepción Global de Estrés (EPGE) diseñada a partir de la comparación lingüística entre la escala adaptada en Chile (Tapia et al., 2007) y México (González \& Landero, 2007b). En estas adaptaciones, se mantienen las catorce preguntas y se autoadministra. Consta de cinco opciones de respuesta para cada pregunta en escala Likert: Nunca, Casi nunca, De vez en cuando, Frecuentemente y Casi siempre. La aplicación del instrumento dura aproximadamente un tiempo de 15 minutos.

Para la evaluación del afecto se utilizó la Escala de Afectos Positivos y Afectos Negativos (SPANAS). El SPANAS evalúa el afecto positivo y negativo (10 ítems de afecto positivo y 10 ítems de afecto negativo). En él se busca medir la intensidad de las experiencias afectivas, mediante una escala Likert que va del 1 (Nunca) al 5 (Siempre). El SPANAS está siendo utilizado en la presente investigación para investigar la validez convergente y divergente del EPGE. En esta investigación se buscará utilizar el SPANAS adaptado por Gargurevich y Matos (2012), en dos muestras de 289 y 175 estudiantes universitarios respectivamente. En esta investigación se hizo un análisis factorial confirmatorio (AFC) de tres modelos (un solo factor, dos factores relacionados y dos factores no correlacionados). 
El modelo que mostró mejores índices de ajuste fue el modelo bifactorial $(\mathrm{S}-\mathrm{BX} 2=856.97, \mathrm{RMSEA}=.68, \mathrm{CFI}=.97, \mathrm{AIC}=938.97)$. La correlación entre las escalas de afecto positivo y negativo fueron positiva para la muestra $1(r=.36, p<.001)$ y la muestra $2(r=.24$, $p<.05)$. Las cargas factoriales para la muestra 1 fueron de .46 a .76 (escala positiva) y de 42. a 91. (escala negativa). Las cargas factoriales para la muestra 2 fueron .50 a .76 (escala positiva) y de .57 a .81 (escala negativa). Sobre la base de su confiabilidad, en la primera muestra, se encuentran coeficientes de consistencia interna para la escala de afecto positivo y negativo de .86 y .90 respectivamente. En la segunda muestra se encuentran coeficientes de consistencia interna para la escala de afecto positivo y negativo de .89 y .90 respectivamente. Los datos de los participantes fueron recogidos en un cuestionario sociodemográfico con el propósito de recolectar datos de los participantes.

\section{Procedimiento}

A fin de obtener la propiedades psicométricas de la EPGE se contactó a los autores de las diversas adaptaciones de la EPGE en países vecinos, cuya lengua es de habla hispana. Se continuó contactando a los encargados del programa de Beca 18 para conseguir la muestra total a aplicar. Para efectos de una evidencia de validez de contenido se comenzó enviando la escala a 11 jueces especialistas, los cuales recibieron una carta en donde se anexaron las instrucciones que debían seguir para realizar una correcta evaluación. Finalmente contestaron 9, habiendo revisado cada uno la plantilla de adaptación lingüística.

Se utilizó la EPGE adaptada en Chile por Tapia et al. (2007) y la adaptada en México por González y Landero (2007b), ya que, según los jueces expertos en comprensión lingüística, están conformadas por un castellano más acorde al peruano y han sido adaptadas también en estudiantes, motivo por el cual definiría una mejor construcción lingüística de cada ítem. Asimismo, se realizó la doble traducción con la escala original de Cohen et al. (1983) con el propósito de mantener los contenidos originales de la prueba. 
Se aplicó la Escala corregida a una muestra piloto de 20 personas de una localidad de escasos recursos de Lima Metropolitana, las edades oscilaban entre los 18 a 30 años de edad. Se eligió esta muestra porque contaba con similitudes en relación con los alumnos del programa de Beca 18, ya que son la gran mayoría migrantes, estudian en la universidad o trabajan, su situación es de probreza o pobreza extrema y hablan en algunos casos el aimara y el quechua. Se volvió a revisar la versión corregida por los jueces siempre en comparación con la versión en original en inglés, para poder visualizar la nueva escala corregida. Posteriormente, se realizó la aplicación a 332 personas del programa Beca 18, previo consentimiento informado aplicado a cada participante, en donde se les explicó la finalidad de la investigación y la confidencialidad que ella guarda.

\section{Análisis de datos}

Se utilizó el programa estadístico SPSS. Se realizó el análisis de las propiedades psicométricas de la Escala y se analizaron las evidencias de validez y confiabilidad de los resultados de ambas pruebas. La validez de la escala se analizó a partir del Análisis Factorial Confirmatorio y la validez convergente y divergente. Para el Análisis Factorial Confirmatorio, se hizo uso del programa estadístico MPLUS versión 7, para efectuar un análisis de los índices de ajuste y ver qué modelo era válido. Valores iguales o inferiores a .05 en el RMSEA sugieren un "buen modelo" (Steiger \& Lind, 1980) y valores entre .051 y .08 en el RMSEA dan evidencia de un "modelo con ajuste admisible" (Ferrando \& Anguiano-Carrasco, 2010).

Por otro lado, puntajes iguales o superiores a .95 en el CFI muestran una buena adecuación del modelo (Gargurevich \& Matos, 2012). Para evaluar la convergencia y divergencia se llevó a cabo la correlación de Spearman, ya que después de realizar la prueba de normalidad de Kolmogorov Smirnov los puntajes de afecto positivo y negativo resultaron menores a .05 , por lo que requiere utilizar en la correlación una prueba no paramétrica entre los puntajes del EPGE y los valores tanto positivos como negativos de la escala SPANAS. En este se plantea como hipótesis que el EPGE debería divergir y converger del SPANAS. 
Para analizar la variabilidad de la puntuaciones, es decir la confiabilidad de la Escala, se empleó el método de consistencia interna utilizando el coeficiente alfa de Cronbach que facilitará el promedio de la correlación entre todos los reactivos que constituyen la Escala. Se debe revisar la correlación de cada ítem con el total del factor, el cual debe ser igual o mayor a .30. El índice de consistencia interna, alfa de Cronbach debe ser al menos .70 para ser considerado aceptable (Alarcón, 2013). Respecto a su interpretación, los autores indican que la escala no es un instrumento diagnóstico, por lo que no cuenta con un punto de corte o baremos. Solamente se pueden realizar comparaciones entre personas de la misma muestra, idealmente para muestras comunitarias.

Por ello se hará un análisis descriptivo que permitirá conocer las diferencias entre variables sociodemográficas, dando así lugar a los resultados finales de la investigación.

\section{Resultados}

Para efectos de una evidencia de validez de contenido se analizaron las respuestas de 9 de los 11 jueces consultados, encontrándose que existía la necesidad de modificar 8 ítems de los catorce que cuenta la escala: el ítem 2, ítem 4, ítem 8, ítem 9, ítem 10, ítem 12 y el ítem 14. Se compararon las observaciones que cada especialista sugirió para cada ítem, especialmente de los ítems 4 y 10, y se modificaron junto con un supervisor especialista en construcción de pruebas psicológicas. Luego de la doble traducción, se utilizaron los ítems 5 y 10 de la escala adaptada en México por González y Landero (2007b). Se agregó a cada pregunta del ítem la frase de "cuán seguido".

Se aplicó la Escala corregida a 20 personas de una localidad de escasos recursos de Lima, Metropolitana, las edades oscilaban entre los 18 a 30 ańos de edad. En las aplicaciones tres de ellos mostraron dificultad en entender el ítem 4, En el último mes, isentiste que enfrentaste exitosamente los cambios importantes que estaban ocurriendo en tu 
vida? catalogándolo como muy largo y confuso y en otras ocasiones como muy redundante o parecido con el ítem 6 el cual se corrigió. Se volvió a revisar la versión corregida por los jueces y se modificó el ítem 4, siempre en comparación con la versión en original en inglés, para poder visualizar la nueva escala corregida.

Posteriormente, se procedió a realizar un Análisis Factorial Confirmatorio para examinar los índices de ajuste. Por ello, para efectos del análisis y comparación de los modelos bifactoriales se tomó en cuenta la oblicuidad de los factores, es decir la correlación de ambos factores, utilizando el método de máxima verosimilitud, para la estimación de los parámetros. A partir de este análisis, se encontró que dentro de los modelos bifactoriales de la EPGE, el modelo de 13 ítems fue el que presentó mejores índices de ajuste. Mientras que el modelo de un solo factor no evidenció un índice de ajuste aceptable (ver Tabla 3).

\section{Tabla 3}

Indices de ajuste de la EPGE

\begin{tabular}{lcccccc}
\hline Modelo & \multicolumn{1}{c}{$\begin{array}{c}\text { Índice de ajuste } \\
\text { absoluto }\end{array}$} & \multicolumn{2}{c}{$\begin{array}{c}\text { Índices de ajuste } \\
\text { de incremento }\end{array}$} \\
& $\chi^{2}$ & $d f$ & p-value & $\begin{array}{c}\text { RMSEA (90\% CI) } \\
\text { TLI }\end{array}$ & CFI \\
\hline $\begin{array}{l}\text { Modelo 2- } \\
\text { Factor EPGE-13 }\end{array}$ & 158.031 & 64 & .000 & $.067(.05-.08)$ & .940 & .951 \\
$\begin{array}{l}\text { Modelo 2- } \\
\text { Factor EPGE-14 }\end{array}$ & 258.367 & 76 & .000 & $.085(.07-.09)$ & .891 & .909 \\
$\begin{array}{l}\text { Modelo 1- } \\
\text { Factor EPGE-14 }\end{array}$ & 531.719 & 65 & .000 & $.147(.136-.159)$ & .709 & .757 \\
\hline
\end{tabular}

Nota: RMSEA=Root Mean Square Error of Approximation (Error de aproximación cuadrático medio), TLI= Tucker-Lewis Coefficient (coeficiente de Tucker y Lewis), CFI= Comparative Fit Index (Índice de ajuste comparativo).

En el modelo bifactorial de 13 ítems las cargas factoriales estandarizadas para el factor de estrés positivo fluctuaron entre .45 y .75; mientras que las cargas factoriales del factor de estrés negativo fluctuaron entre .36 y .72 (ver Figura 1). Dicho resultado confirma que los 
datos de la muestra del presente estudio ajustan de manera adecuada a una estructura bifactorial, en donde se puede encontrar por un lado un factor en relación al estrés negativo (6 ítems) y un factor de estrés positivo (7 ítems).

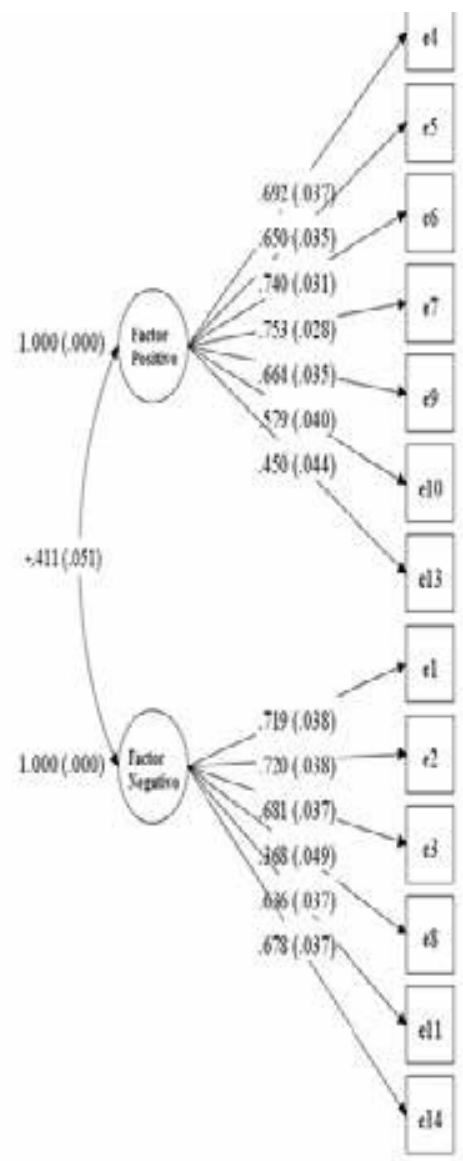

Figura 1. Modelo de 2-Factores de la EPGE-1 
Una vez realizados los análisis de ACP y AFC, se pasó a realizar las evidencias de validez convergente y divergente de la EPGE utilizando la prueba SPANAS. Se inició examinando la distribución de los puntajes obtenidos en la muestra en la presente investigación utilizando el test de Kolmogorov-Smirnov. En la Escala de Percepción Global de Estrés se evidenció que el factor de eustrés $(Z=.085, p=.00)$ y el factor de distrés $(Z$ $=.071, p=.00)$ no se encontraban distribuidos normalmente. De igual manera, sucedió entre los factores de afecto positivo $(Z=.085, p=.00)$ y afecto negativo $(Z=.080, p=.00)$ en el SPANAS. Tomando en cuenta estos resultados se realizó el análisis de correlación de Spearman debido a que ninguna de las variables intervinientes se encontraban distribuidas normalmente por lo que se debian de utilizar estadisticos no paramétricos.

Las evidencias de validez convergente y divergente de las escalas fueron evaluadas a partir de los resultados de las correlaciones entre los factores de eustrés — estrés positivo-y distrés — estrés negativode la EPGE con los factores de afecto positivo y afecto negativo del SPANAS (ver Tabla 4).

\section{Tabla 4}

Correlaciones de Spearman entre la EPGE y el SPANAS

\begin{tabular}{lcccc}
\hline Variables & 1 & 2 & 3 & 4 \\
\hline 1. EPGE Negativo & - & & & \\
2. EPGE Positivo & $-.41^{* *}$ & - & & \\
3. SPANAS Negativo & $.60^{* *}$ & $-.39^{* *}$ & - & \\
4. SPANAS Positivo & $-.24^{* *}$ & $.39^{* *}$ & $-.14^{* *}$ & - \\
\hline
\end{tabular}
${ }^{* *} p<.01$

Los resultados mostraron que entre los factores de eustrés y distrés se obtuvo una correlación negativa significativa $(r=-.411, p<.00)$. Al analizar la convergencia entre ambas escalas se encontró que el factor de distrés de la EPGE correlacionó de manera positiva y significativa con el factor de afecto negativo $(r=.597, p<0.000)$, Asimismo el factor de eustrés de la EPGE correlacionó de manera positiva y significativa con el factor de afecto positivo $(r=.387, p<0.000)$. Al analizar la 
divergencia entre ambas escalas se encontró que el factor de distrés de la EPGE correlacionó de manera negativa y significativa con el factor de afecto positivo $(r=-.242, p<0.000)$, Asimismo el factor de eustrés de la EPGE correlacionó de manera negativa y significativa con el factor de afecto negativo $(r=-.393, p<0.000)$.

Para analizar la confiabilidad de la escala se empleó el método de consistencia interna de alfa de Cronbach. En el caso de los ítems que componen el factor de eustrés, el índice de consistencia interna de alfa de Cronbach fue de .799, con la correlación ítem total corregida fluctúa entre .41 y .60. Para el factor de distrés el índice de consistencia interna de alfa de Cronbach fue de .770, con la correlación ítem total corregida que fluctúa entre 32 y .59 .

Debido a que el EPGE no tiene un puntaje de corte (Tapia et al., 2007) la obtención de este se calculó estadísticamente en base a los resultados obtenidos en esta investigación. De esta manera se dividieron los puntajes totales de cada dimensión en los siguientes percentiles: el $\mathrm{P}_{25}$ para un nivel bajo, que va desde 0 a 9 puntos en el distrés y de 0 a 17 puntos en el eustrés; el $\mathrm{P}_{50}$ para un nivel Moderado, que va desde un puntaje de 10 hasta 12 para el distrés y de 10 hasta 22 en el eustrés; y un $\mathrm{P}_{75}$ para un nivel alto, que va desde 13 hasta 24 en distrés y de 23 hasta 28 en eustrés. En base a estos parámetros se encontró que el 48.8\% $(n=162)$ de la muestra total presenta un nivel alto Estrés Negativo. En contraparte, sobre la base del Estrés Positivo, un $18.1 \%(n=60)$ presentan un nivel alto.

En los puntajes del EPGE según sexo se encontró que en los niveles de eustrés existen diferencias significativas, $x^{2}(2)=15.733$, $p<.001$. Esto se presenta en la diferencia del número de personas que presentan niveles altos de eustrés, según hombres y mujeres. Por un lado, el $28.3 \%$ de las mujeres $(n=17)$, presentan un nivel alto de eustrés en comparación con los hombres en donde un $71,7 \%(n=43)$ presentan un nivel alto de eustrés. Dentro de los niveles de distrés al igual que los resultados de eustrés, se encontraron diferencias significativas, $x^{2}(2)=17.468, p<.001$. Estas diferencias se pueden ver entre el número de personas que presentan niveles altos de distrés, según hombres y mujeres. Por un lado, el $62.3 \%$ de las mujeres $(n=101)$, presentan un 
nivel alto de distrés en comparación con los hombres en donde un $37.7 \%(n=61)$ presentan un nivel alto de eustrés (ver Tabla 5).

\section{Tabla 5}

Niveles altos de eustrés y distrés según sexo

\begin{tabular}{llccc}
\hline \multirow{2}{*}{ Niveles altos de estrés } & & \multicolumn{2}{c}{ Sexo } & \multirow{2}{*}{ Total } \\
\cline { 3 - 4 } & & Femenino & Masculino & \\
\hline $\begin{array}{l}\text { Estrés positivo } \\
\text { (eustrés) }\end{array}$ & Recuento & 17 & 43 & 60 \\
& $\%$ & 28,3 & $71.7 \%$ & 100 \\
$\begin{array}{l}\text { Estrés negativo } \\
\text { (distrés) }\end{array}$ & Recuento & 101 & 61 & 165 \\
& $\%$ & 62.3 & $37,7 \%$ & 100 \\
\hline
\end{tabular}

Respecto a los resultados obtenidos en el EPGE y la variable lugar de procedencia, en los niveles de eustrés, no existen diferencias significativas entre los alumnos que provienen de Lima y los que provienen del interior del país, $x^{2}(2)=2.822, p<.244$. Asimismo, dentro de los niveles de distrés, no existen diferencias significativas entre los alumnos que provienen de Lima y los que provienen del interior del país, $x^{2}(2)=$ $1.063, p<.588$. Sin embargo, existe una similitud entre el gran número de alumnos que presentan niveles altos de distrés y el bajo número de alumnos que presentan niveles altos de eustrés (ver Tabla 6).

\section{Tabla 6}

Niveles altos de eustrés y distrés según lugar de procedencia

\begin{tabular}{llcc}
\hline \multirow{2}{*}{ Lugar de procedencia } & & \multicolumn{2}{c}{ Niveles del estrés } \\
\cline { 3 - 4 } & & Nivel alto eustrés & Nivel alto distrés \\
\hline Lima $(n=72)$ & Recuento & 11 & 39 \\
& $\%$ & 15.27 & 54.16 \\
Interior del país & Recuento & 49 & 130 \\
$(n=260)$ & $\%$ & 18.84 & 47.30 \\
\hline
\end{tabular}




\section{Discusión}

El objetivo de la presente investigación consistió en examinar las evidencias de validez y confiabilidad de la Escala de Percepción Global de Estrés de Cohen et al. (1983) en una muestra de alumnos universitarios de Beca 18. Los resultados presentaron que la Escala de Percepción Global de Estrés evidenció una adecuada validez interna y externa, a partir de un análisis de contenido y un Análisis Factorial Confirmatorio, y evidencias de convergencia y divergencia respectivamente, así como una adecuada confiabilidad estimada de los puntajes. El hecho de haber aplicado la prueba a personas provenientes del interior del país, cuya comprensión del castellano no es del todo clara, asegura que el castellano de los ítems que resultaron de la revisión de jueces, comparación lingüística y doble traducción fueron los adecuados para su comprensión por parte de la muestra. Por ello se puede decir que la escala cuenta con una facilidad de comprensión, asegurando la rapidez en su aplicación y su practicidad para el uso diario en la práctica clínica y de investigación.

En la validez de constructo se encontró que la Escala cuenta con una estructura bifactorial, lo cual confirma la estructura obtenida en la versión de Cohen \& Williamson (1988) y con el resto de las adaptaciones a nivel internacional (Andreou et al. 2011; Benítez et al., 2012; González \& Landero, 2007b; Mimura \& Griffiths, 2004; Leung, Lam \& Chan, 2010; Remor, 2006; Stauder \& Konkoly, 2006; Tapia et al. 2007). Asimismo, el Análisis Factorial Confirmatorio presentó que el modelo de dos factores correlacionados sin el ítem 12 era el que mejor representó a la escala en la muestra. Algunos autores interpretan que estos dos factores son el reflejo dos dimensiones: la de percepción de desamparo y la de auto eficacia percibida (Orucu \& Demir, 2008).

Por otro lado, para otros autores, el factor positivo es un efecto de redacción, considerado la escala como unidimensional (Lesage, Berjot \& Deschamps, 2012). Sin embargo para efectos de este estudio decidimos mantener la Escala conformada con dos subescalas, una positiva y otra negativa, para que al momento de sacar los resultados podamos 
visualizar en los alumnos en qué aspectos están reflejando una adecuada capacidad de lidiar con el estrés existente (ítems positivos), los cuales pueden ser resaltados o repotenciados, en caso de que estén bajos; y en qué aspectos están reflejando una incapacidad de autocontrol (ítems negativos) ante situaciones generadoras de estrés (Hewitt, Flett \& Mosher, 1992).

Con relación a adaptaciones anteriores como las realizadas por Benítez et al. (2012), en Venezuela, en donde el ítem 12 fue eliminado; y la realizada por Tapia et al. (2007), en donde el ítem 10 pasó de ser un ítem positivo a uno negativo, la EPGE eliminó el ítem 12 de la dimensión de estrés negativo, resultando la escala con 13 ítems originales. Esto se dio ya que su carga factorial era baja $(<0.4)$ y muy similar en ambos factores. Esta ambivalencia pudo haber sido resultado de la interpretación por parte del alumno, en el cómo se comprendía el ítem 12: "En el último mes ¿Cuán seguido te has encontrado pensando en las cosas que tienes que lograr?". Para un alumno del programa Beca 18 la planificación de su tiempo de trabajo, estudio y descanso es vital para obtener buenas notas y mantener su beca vigente, por lo que es en cierto sentido necesario que se encuentren pensando en las cosas que tienen que lograr a manera de objetivos, para cumplirlos y mantener esta gran posibilidad de estudios superiores. Sin embargo, esto no quita el hecho de que el estar pensando constantemente en ello no vayan a presentar un estrés que puede empezar en positivo, y de no sobrellevarlo, se frustre y se transforme en uno negativo. Por ello, vemos que dicha ambivalencia hace que el ítem 12 no se acople a la situación real en la que viven estos jóvenes y por ello su exclusión de la escala (Anexo 3).

En cuanto a la validez convergente y divergente, las correlaciones con las medidas de afecto positivo y negativo del SPANAS fueron adecuadas, apoyando su uso en la muestra evaluada. La convergencia y divergencia entre la EPGE y el SPANAS indica que cuando una persona se encuentra frente a situaciones de estrés las cuales no puede controlar o sobrellevar de forma adaptativa, una serie de reacciones de afecto negativo como la ira, decepción o nerviosismo pueden estar presentes, 
de igual modo en el caso de situaciones en las que la persona presenta la capacidad de sobrellevar un hecho generador de estrés (Hewitt et al., 1992).

La confiabilidad estimada de los puntajes generados por el instrumento demostró que cada uno de sus dos factores cuenta con una fiabilidad aceptable $(\alpha>.70)$. Esto indica que los ítems miden un mismo constructo (negativo y positivo) y que están correlacionados (Oviedo \& Campo-Arias, 2005). En adaptaciones anteriores los coeficientes de alfa de Cronbach varían entre aceptables y buenos. Los coeficientes de consistencia interna del factor negativo y positivo de la EPGE se encuentran dentro de lo aceptable $(\alpha>.70)$, y guardan similitud con el resto de las adaptaciones que tienen coeficientes de consistencia interna dentro de lo aceptable (Andreou et al. 2011; González \& Landero, 2007b; Lesage, Berjot \& Deschamps, 2012; Leung, Lam \& Chan, 2010; Mimura \& Griffiths, 2004). Esto puede deberse a varios puntos: a la poca variabilidad entre los sujetos que se estudiaron en cada estudio, al tamaño de la escala que mantuvo casi toda la totalidad de sus ítems y a las adecuadas adaptaciones lingüísticas que se han hecho en cada adaptación, lo cual han facilitado que los ítems se respondan correctamente.

Por otro lado, se puede decir que los resultados en base al estrés percibido según sexo, el puntaje de las mujeres sigue manteniéndose por encima del puntaje obtenido por los hombres (Andreou et al. 2011; Benítez et al., 2012; Cohen et al., 1983; González \& Landero, 2007b; Remor, 2006; Tapia et al., 2007). Estos resultados pueden estar obedeciendo al hecho de que existe una dificultad en las mujeres de encontrar recursos personales que le permitan controlar la amenaza percibida en su entorno. Esto se refiere a que según la teoría del modelo transaccional del estrés existe un problema en la valoración secundaria en donde se tendría que buscar más si dicha incapacidad de conseguir un recurso adecuado está siendo generado por problema de corte social, material, psicológico o físico (Arenas \& Puigcerver, 2009; Valls, 1994). Por otro lado, autores como Madden, Feldman y Pietromonaco (2000), postulan que las mujeres expresan con mayor facilidad los sen- 
timientos de tristeza, malestar y miedo lo cual hace que ante los ítems del factor negativo obtengan mayor puntaje (Lee, 2012).

Es importante recalcar que no existió diferencia significativa entre el estrés percibido en alumnos migrantes y alumnos de Lima. Sin embargo, es importante recalcar los niveles altos de las dimensiones negativas. Esto nos lleva a concluir que la mayoría de los alumnos al no tener un alto nivel de eustrés que les ayude a afrontar adecuadamente una situación estresora, se encuentran vulnerables a padecer un mayor estrés desadaptativo que les impida desenvolverse adecuadamente en lo académico. Por ello es importante ver qué parte del programa, en conjunto con las universidades, no está siendo llevado a cabo en pro a las necesidades sociales, personales o familiares que desarrollan una adecuada resiliencia para incrementar su autoestima, percepción de autoeficacia, en sus expectativas de éxito, optimismo y esperanza (Garmezy, 1993; Richardson, 2002; Rutter, 1987).

Finalmente, en el caso de los alumnos del interior del país este resultado puede estar obedeciendo a los factores psicosociales (Mcgrath, 1970; Molina-Jiménez, Gutierrez-García, Hernández-Dominguez $\&$ Contreras, 2008); que se presentan cuando un migrante sufre los duelos de abandonar una familia, una localidad o hasta sus costumbres, los cuales pueden, en relación con estresores del entorno, llegar a desencadenar, si es que no se realiza una intervención de adecuada, el Síndrome de Ulises (Achotegui, 2010). Este padecer de no detectarse e intervenirlo, impediría al alumno el poder adaptarse a su nuevo entorno, repercutiendo en lo académico y con ello en su beca.

En cuanto a la limitación de esta investigación estuvo relacionada con los participantes. En esta investigación, los participantes son todos estudiantes universitarios del programa Beca 18, lo cual solo representa a un sector de la población del país, y no se extiende a otros ámbitos como el de la salud y laboral, como las adaptaciones de Remor (2006) en España o Cohen et al. (1983) en Estados Unidos. 


\section{Referencias}

Achotegui, J. (2010). Cómo evaluar el estrés y el duelo migratorio (2a. Ed.). Barcelona, España: El mundo de la mente.

Alarcón, R. (2013). Métodos y Diseños de investigación del comportamiento. Lima, Perú: Universidad Ricardo Palma Editorial Universitaria.

Andreou, E., Alexopoulos, E. C., Lionis, C., Varvogli, L., Gnardellis, C., Chrousos, G.P. \& Darviri, A. (2011). Perceived Stress Scale: Reliability and Validity study un Greece. International Journal of Enviromental Research and Public Health, 8, 3287-3298. https:// doi.org/10.3390/ijerph8083287

Arenas, M. \& Puigcerver, A. (2009). Diferencias entre hombres y mujeres en los trastornos de ansiedad: una aproximación psicobiológica. Escritos de Psicología, 3, 1, 20-29.

Barraza, A., Carrasco, R. \& Arreola, M. (2009). Burnout Estudiantil. Un estudio exploratorio. Trabajo presentado en el $\mathrm{X}$ Congreso Nacional de Investigación educativa, Veracruz, Veracruz. Recuperado de: http://www.comie.org.mx/congreso/memoriaelectronica/v10/pdf/area_tematica_16/ponencias/0614-F.pdf

Barraza, A. (2006). Un modelo conceptual para el estudio de estrés académico. Revista electrónica de psicología de Iztacala, 9(3), 110-129.

Benítez, A., D’Anello, S. \& Noel, G. (2012). Validación de la escala de estrés percibido (EEP-13) en una muestra de funcionarios públicos venezolanos. Anales de la Universidad Metropolitana, 13, 117-133.

Berrio, N. \& Mazo, R. (2011). Caracterización psicométrica del inventario de estrés académico en estudiantes de pregrado de la universidad de Antioquía. (Trabajo de grado para optar al título de psicología). Colombia: Universidad de Antioquía.

Cohen, S. \& Williamson, G. (1988). Perceived Stress in a Probability Sample of the United States. The Social Psychology of Health, 3, 31-67. 
Cohen S. T., Kamarck, R. \& Mermelstein, R. (1983). A Global Measure of Perceived Stress. Journal of Health and Social Behavior, 24, 385-396. https://doi.org/10.2307/2136404

Emerson, E. (2004). Poverty and Children with intellectual disabilities in the world richest countries. Journal of intellectual \& Development Dissability, 29(4), 319-338. https://doi. org/10.1080/13668250400014491

Ferrando, P. \& Anguiano-Carrasco, C. (2010). El análisis factorial como técnica de Investigación en Psicología. Papeles del Psicó$\log 0,31(1), 18-33$.

Gallardo, G. \& Morales, Y. (2011). La universidad para el aprendizaje de todos. Santiago de Chile: Pontificia Universidad Católica de Chile.

García-Ros, R., Pérez-González, F., Pérez-Blasco, J. \& Natividad, L. (2011). Evaluación del estrés académico en estudiantes de nueva incorporación en la universidad. Revista Latinoamericana de Psicología, 44, 143-154.

Gargurevich, R. \& Matos, L. (2012). Validez y Confiabilidad de la escala de afecto positivo y negativo (SPANAS) en estudiantes universitarios peruanos. Revista Psicológica, 14(2), 208-217.

Garmezy, N. (1993). Children in poverty: Resilience despite risk. Psychiatry, 56, 127-136. https://doi.org/10.1080/00332747.19 93.11024627

González, M.T \& Landero, R. (2007a). Escala de cansancio emocional (ECE) para estudiantes universitarios: Propiedades psicométricas de una muestra de México. Anales de Psicología, 23(2), 253-257. González, M.T \& Landero, R. (2007b). Factor Structure of the Perceived Stress Scale (PSS) in a Sample from Mexico. The Spanish Journal of Psychology, 10, 199-206. https://doi.org/10.1017/ S1138741600006466

Hewitt, P., Flett, G. \& Mosher, S. (1992). The Perceived Stress Scale: Factor Structure and Relation to Depression Symptom in a Psychiatric Sample. Journal of Psychopatology and Behavioral Assesment, 14(3), 247-257. https://doi.org/10.1007/BF00962631 
Instituto Nacional de Estadística e Informática (2015). Informe Técnico: Evolución de la pobreza monetaria 2009-2014. Lima, Perú.

Lazarus, R. \& Folkman, S. (1986). Estrés y procesos cognitivos. Barcelona, España: Ediciones Martínez Roca.

Lee, E. (2012). Review of Psychometric Evidence of the Perceived Stress Scale. Asian Nursing Research, 6(4), 121-127. http:// dx.doi.org/10.1016/j.anr.2012.08.004

Lee, E., Chung, B., Suh, C. \& Jung, J. (2015). Korean version of the Perceived Stress Scale (PSS-14, 10 and 4): psychometric evaluation in patients with chronic disease. Scandinavian Journal of Caring Science, 29, 183-192. https://doi.org/10.1111/ scs. 12131

Lera, M. (2009). Impacto de la pobreza crónica en la resiliencia: claves para entender la perpetuidad de la miseria. Paideia, 46, 73-97.

Lesage, F., Berjot, S. \& Deschamps, F. (2012). Psychometric Properties of the French Versions of the Perceived Stress Scale. International Journal of the Occupational Medicine and Environmental Health, 25, 178-184. https://doi.org/10.2478/s13382-012-0024-8

Leung, D., Lam, T. \& Chan, S. (2010). Three versions of Perceived Stress Scale: Validation in sample of Chinese cardiac patients who smoke. BioMed Central Public Health, 10, 513. https://doi. org/10.1186/1471-2458-10-513

López, J., Ato, M. \& Benavente, A. (2013). Un sistema de clasificación de los diseños de investigación en psicología. Anales de Psicología, 29(3), 1038-1059. http://dx.doi.org/10.6018/ analesps.29.3.178511

Madden, T., Feldman, L. \& Pietromonaco, P. (2000). Sex difference in anxiety and depression: Empirical evidence and methodological questions. En A. Fisher (Eds.), Gender and Emotion (pp. 277298). Cambridge: Cambridge University Press. https://doi. org/10.1017/CBO9780511628191.014

Mcgrath, J. (1970). Social and psychological factors in stress. Nueva York, NY: Rinehart and Winston. 
Mimura, A. \& Griffiths, P. (2004). A Japanese version of the perceived stress scale: Translation and Preliminary Test. International Journal of Nursing Studies, 41, 379-385. https://doi. org/10.1016/j.ijnurstu.2003.10.009

Molina-Jiménez, T., Gutierrez-García, A., Hernández-Dominguez, L. \& Contreras, C. (2008). Estrés psicosocial: algunos aspectos clínicos y experimentales. Anales de Psicología, 24, 353-360.

Orucu, M. \& Demir, A. (2008). Psychometric evaluation of perceived stress scale for Turkish university students. Stress and Health, 25, 103-109. https://doi.org/10.1002/smi.1218

Oviedo, C. \& Campo-Arias, A. (2005). Aproximación al uso del coeficiente alfa de Cronbach. Revista Colombiana de Psiquiatría, 34(4), 572-580.

Programa Nacional de Becas y Crédito Educativo. (2014). Bases del concurso para el otorgamiento de Beca 18 de pregrado convocatoria 2014. Recuperado de http://www.pronabec.gob.pe/inicio/ becas/descargas/bases_beca182014.pdf

Pulido, M., Serrano, M., Cano, E., Chavez, M., Montiel, P. \& Vera, F. (2011). Estrés académico en estudiantes universitarios. Psicologia y Salud, 21, 31-37.

Remor, E. (2006). Psychometric Properties of a European Spanish Version of the Perceived Stress Scale (PSS). The Spanish Journal of Psychology, 9, 86-93. https://doi.org/10.1017/ S1138741600006004

Richardson, G. (2002). The metatheory of resilience and resiliency. Journal of Clinical Psychology, 58(3), 307-321. https://doi. org/10.1002/jclp. 10020

Rutter, M. (1987). Psychosocial resilience and protective mechanisms. American Journal of Orthopsychiatry, 57, 316-331. https://doi. org/10.1111/j.1939-0025.1987.tb03541.x

Stauder, A. \& Konkoly, B. (2006). Characteristics of the Hungarian version of the Perceived Stress Scale (PSS). Mentálhigiéné és Pszichoszomatica, 7(3), 203-216. https://doi.org/10.1556/ Mental.7.2006.3.4 
Steiger, J. \& Lind, J. (Mayo, 1980). Statistically-based tests for the number of common factors. Trabajo presentado en la Annual Spring Meeting of the Psychometric Society, Iowa.

Tapia, D., Cruz, C., Gallardo, I. \& Dasso, M. (2007). Adaptación de la Escala de Percepción Global de Estrés - PSS (Cohen, Kamarck $\&$ Mermelstein, 1983), en una población de adultos de escasos recursos que estudian carreras técnicas en la comuna de San Joaquín, Región Metropolitana, Chile. Psiquiatría y Salud Mental, 24, 109-119.

Trianes, M., Blanca, M., Fernández-Baena, J., Escobar, M. \& Maldonado, E. (2012). Evalución y tratamiento del estrés cotidiano en la infancia. Papeles del Psicólogo, 33(1), 30-45.

Valls, C. (1994). Consecuencias orgánicas del estrés. Cómo afecta de forma diferenciada a hombres y mujeres. En Jornada 5. Incidencia del estrés en la salud de las mujeres. Vitoria-Gasteiz, España: Emakunde/Instituto Vasco de la Mujer.

Wadsworth, M. E., Raviv, T., Reinhard, C., Wolff, B., Santiago, C. D. \& Einhorn, L. (2008). An Indirect Effects Model of the Association Between Poverty and Child Functioning: The Role of Children's Poverty-Related Stress. Journal of Loss and Trauma, 13(2), 156-185. https://doi.org/10.1080/15325020701742185

Recibido: 16 de enero, 2017

Revisado: 23 de mayo, 2018

Aceptado: 28 de mayo, 2018 


\section{Anexo 1}

\section{Adaptaciones de la EPGE en Asia}

\begin{tabular}{|c|c|c|c|c|}
\hline Referencia & Población & $\begin{array}{l}\text { Alfa de } \\
\text { Cronbach }\end{array}$ & $\begin{array}{l}\text { Confiabilidad } \\
\text { por Test retest }\end{array}$ & Validez \\
\hline $\begin{array}{l}\text { Leung, } \\
\text { Lam \& } \\
\text { Chan } \\
(2010)\end{array}$ & $\begin{array}{c}\text { Pacientes } \\
\text { cardiacos que } \\
\text { fuman }(N= \\
1800)\end{array}$ & $\begin{array}{c}.86 \text { y } .77 \text { para } \\
\text { los factores } \\
\text { positivos y } \\
\text { negativos } \\
\text { respectiva- } \\
\text { mente. }\end{array}$ & - & $\begin{array}{c}\text { Análisis Factorial } \\
\text { Confirmatorio } \\
\text { indica un ajuste } \\
\text { adecuado. } \\
\text { Validez } \\
\text { convergente: } \\
\text { FTND ( } r= \\
\text {.11), Confianza } \\
\text { en no volver a } \\
\text { fumar ( } r=-.19), \\
\text { Estado Saludable } \\
\text { Percibido }(r= \\
\text {-.16), Ansiedad ( } r \\
=.18) \text { y Depresión } \\
(r=.22)\end{array}$ \\
\hline $\begin{array}{l}\text { Mimura } \\
\qquad \& \\
\text { Griffiths } \\
(2004)\end{array}$ & $\begin{array}{l}\text { Estudiantes de } \\
\text { un programa } \\
\text { de Post grado: } \\
\text { japoneses } \\
(n=23) \text { e } \\
\text { ingleses }(n=38)\end{array}$ & $\begin{array}{l}.81-.84 \text { para } \\
\text { los factores } \\
\text { positivos y } \\
\text { negativos } \\
\text { respectiva- } \\
\text { mente. }\end{array}$ & - & $\begin{array}{l}\text { Análisis Factorial } \\
\text { Eploratorio con } \\
\text { rotación varimax: } \\
\text { estructura } \\
\text { bifactorial } \\
\text { representó el } \\
50-53.2 \% \text { de la } \\
\text { varianza) }\end{array}$ \\
\hline $\begin{array}{c}\text { Lee et al. } \\
(2015)\end{array}$ & $\begin{array}{l}\text { Personas de } \\
\text { un hospital } \\
\text { universitario y } \\
\text { centro de salud } \\
\quad(N=402)\end{array}$ & $\begin{array}{c}.87 \text { y } .85 \text { para } \\
\text { los factores } \\
\text { negativos y } \\
\text { positivos, } \\
\text { respectiva- } \\
\text { mente. }\end{array}$ & $\begin{array}{c}I C C=.80 \text { por } \\
\text { un intervalo de } \\
\text { una semana. } \\
70.29 \% \\
\text { completaron la } \\
\text { reaplicación. }\end{array}$ & $\begin{array}{c}\text { Validez } \\
\text { concurrente: } r= \\
.63 \text { con el CES-D }\end{array}$ \\
\hline
\end{tabular}

Nota: FTND= Fagerstrom Test of Nicotine Dependency; CES-D = Center for Epidemiologic Studies-Depression 


\section{Anexo 2}

\section{Adaptaciones de la EPGE en Europa}

\begin{tabular}{|c|c|c|c|c|}
\hline Referencia & Población & $\begin{array}{l}\text { Alfa de } \\
\text { Cronbach }\end{array}$ & $\begin{array}{l}\text { Confiabilidad } \\
\text { por Test retest }\end{array}$ & Validez \\
\hline $\begin{array}{l}\text { Lesage et } \\
\text { al. (2012) }\end{array}$ & $\begin{array}{l}\text { Trabajadores } \\
(N=501)\end{array}$ & $\begin{array}{l}.79 \text { y. } 81, \\
\text { negativo y } \\
\text { positivo, } \\
\text { respectiva- } \\
\text { mente. }\end{array}$ & - & $\begin{array}{c}\text { Validez Factorial: } \\
\text { con Rotación } \\
\text { Oblimin se } \\
\text { encontró una } \\
\text { estructura de } \\
\text { dos factores } \\
\text { que explicaban } \\
\text { el } 49 \% \text { de la } \\
\text { varianza. }\end{array}$ \\
\hline $\begin{array}{l}\text { Andreou } \\
\text { et al. } \\
(2011)\end{array}$ & $\begin{array}{l}\text { Personas } \\
\text { de centro } \\
\text { de salud, } \\
\text { educativos } \\
\text { y oficinas } \\
\text { financieras } \\
(N=941)\end{array}$ & $\begin{array}{l}.79 \text { y. } 77, \\
\text { negativo y } \\
\text { positivo, } \\
\text { respectiva- } \\
\text { mente. }\end{array}$ & - & $\begin{array}{c}\text { Correlación } \\
\text { moderada con el } \\
\text { DASS-21. } \\
\text { Validez Factorial: } \\
\text { se econtró } \\
\text { una estructura } \\
\text { bifactorial en la } \\
\text { EPGE. }\end{array}$ \\
\hline $\begin{array}{l}\text { Stauder \& } \\
\text { Konkoly } \\
(2006)\end{array}$ & $\begin{array}{l}\text { Personas de } \\
\text { un programa } \\
\text { para el control } \\
\text { de estrés } \\
(N=217)\end{array}$ & .88 & $\begin{array}{l}r=.90 \text { por un } \\
\text { intervalo de } 5 \\
\text { días. }\end{array}$ & $\begin{array}{c}\text { Validez } \\
\text { convergente: } \\
r=.81 \text { con el } \\
\text { STAI-T }\end{array}$ \\
\hline
\end{tabular}

Nota: DASS-21 = Depression Anxiety Stress Scale-21; EPGE=Escala de Percepción Global de Estrés;STAI-T = State-Trait Anxiety Inventory-Trait 


\section{Anexo 3}

Escala de Percepción Global de Estrés para Universitarios (Adaptado en estudiantes universitarios peruanos)

\begin{tabular}{|c|c|c|c|c|c|}
\hline \multicolumn{6}{|c|}{$\begin{array}{l}\text { Marca con una "X" en el casillero aquella opción que exprese mejor tu situación actual, } \\
\text { teniendo en cuenta el último mes. Para cada pregunta coloca solo una opción. }\end{array}$} \\
\hline & Nunca & $\begin{array}{c}\text { Casi } \\
\text { nunca }\end{array}$ & $\begin{array}{c}\text { De vez en } \\
\text { cuando }\end{array}$ & $\begin{array}{c}\text { Frecuente- } \\
\text { mente }\end{array}$ & $\begin{array}{c}\text { Casi } \\
\text { siempre }\end{array}$ \\
\hline $\begin{array}{l}\text { 1. En el último mes ¿Cuán seguido has estado } \\
\text { molesto por que algo pasó de forma inesperada? }\end{array}$ & & & & & \\
\hline $\begin{array}{l}\text { 2. En el último mes ¿Cuán seguido te has sentido } \\
\text { incapaz de controlar hechos importantes en tu vida? }\end{array}$ & & & & & \\
\hline $\begin{array}{l}\text { 3. En el último mes ¿Cuán seguido te has sentido } \\
\text { continuamente tenso? }\end{array}$ & & & & & \\
\hline $\begin{array}{l}\text { 4. En el último mes ¿Cuán seguido te sentiste } \\
\text { seguro de tus habilidades para manejar tus } \\
\text { problemas personales? }\end{array}$ & & & & & \\
\hline $\begin{array}{l}\text { 5. En el último mes ¿Cuán seguido has sentido } \\
\text { que has afrontado efectivamente los cambios } \\
\text { importantes que han estado ocurriendo en tu vida? }\end{array}$ & & & & & \\
\hline $\begin{array}{l}\text { 6. En el último mes ¿Cuán seguido confiaste } \\
\text { en tu capacidad para manejar tus problemas } \\
\text { personales? }\end{array}$ & & & & & \\
\hline $\begin{array}{l}\text { 7. En el último mes ¿Cuán seguido sentiste que } \\
\text { las cosas te estaban resultando como tú querías? }\end{array}$ & & & & & \\
\hline $\begin{array}{l}\text { 8. En el último mes ¿Cuán seguido te diste } \\
\text { cuenta que no podías hacer todas las cosas que } \\
\text { debías hacer? }\end{array}$ & & & & & \\
\hline $\begin{array}{l}\text { 9. En el último mes ¿Cuán seguido has podido } \\
\text { controlar las dificultades de tu vida? }\end{array}$ & & & & & \\
\hline $\begin{array}{l}\text { 10. En el último mes ¿Cuán seguido has sentido } \\
\text { que tienes el control de todo? }\end{array}$ & & & & & \\
\hline $\begin{array}{l}\text { 11. En el último mes ¿Cuán seguido te has } \\
\text { sentido molesto por situaciones que estaban fuera } \\
\text { de tu control? }\end{array}$ & & & & & \\
\hline $\begin{array}{l}\text { 12. En el último mes ¿Cuán seguido pudiste } \\
\text { controlar la manera en que utilizaste el tiempo? }\end{array}$ & & & & & \\
\hline $\begin{array}{l}\text { 13. En el último mes ¿Cuán seguido sentiste que } \\
\text { los problemas se te habían acumulado? }\end{array}$ & & & & & \\
\hline
\end{tabular}

